\title{
Review and Prospect of Research on Floating Population Entrepreneurship in Strange Land in China
}

\author{
Ke Ying \\ School of Management, Jinan University, Guangzhou, China \\ Email: keying3643@163.com
}

How to cite this paper: Ying, K. (2020). Review and Prospect of Research on Floating Population Entrepreneurship in Strange Land in China. American Journal of Industrial and Business Management, 10, 659-676. https://doi.org/10.4236/ajibm.2020.103044

Received: March 2, 2020

Accepted: March 21, 2020

Published: March 24, 2020

Copyright (๑) 2020 by author(s) and Scientific Research Publishing Inc. This work is licensed under the Creative Commons Attribution International License (CC BY 4.0).

http://creativecommons.org/licenses/by/4.0/

\section{(c) (i) Open Access}

\begin{abstract}
With the development of urbanization in China, the floating population has become an important driving force for urban economic development and entrepreneurial activity, and its entrepreneurial activity even exceeds that of local residents. This paper focuses on the main theoretical results of the research on floating population entrepreneurship in strange land, systematically combs and reviews the research on floating population entrepreneurship in strange land from three aspects: the definition and measurement of floating population entrepreneurship in strange land, the determinants of floating population entrepreneurship in strange land, and the results of floating population entrepreneurship in strange land. Floating population entrepreneurship in strange land refers to the behavior of floating population to identify, create and utilize economic opportunities, carry out entrepreneurial activities and achieve enterprise development in their inflow areas. In addition to focusing on the entrepreneurial behavior of the general floating population, the research on floating population entrepreneurship in strange land also focuses on the urban entrepreneurial behavior of the rural floating population. The research on the determinants of floating population entrepreneurship in strange land mainly focuses on the single factor analysis at the micro, meso and macro levels. The research on the results of floating population entrepreneurship in strange land explores the multiple impacts of floating population entrepreneurship on entrepreneurs and entrepreneurial regions from an economic and non-economic perspective. Finally, based on the existing analysis conclusions and deficiencies, this paper expands the future research direction. This paper considers that the subdivision of types of floating population entrepreneurship in strange land, the interaction of determinants of floating population entrepreneurship in strange land, the process research of floating population entrepreneurship in strange land, and the entrepreneurial
\end{abstract}


performance of floating population in strange land need to be further deepened.

\section{Keywords}

Floating Population, Rural Floating Population, Entrepreneurship in Strange Land

\section{Introduction}

In China, the discrimination and exclusion caused by the household registration system have led to the widespread phenomenon of unequal pay for equal work between floating population and local residents in terms of wage income (Zhang, 2016). It is difficult for floating population to improve their economic and social status through promotion in the labor market. Early studies on floating population mainly focused on the social security, social integration and social crime, and paid less attention to the positive social behavior of floating population. In recent years, some studies have found that urban entrepreneurship is the effective way for floating population to make their living and improve economic and social status, and the entrepreneurial intention and entrepreneurial rate of floating population is even higher than that of local residents (Liu \& Huang, 2016; Liu et al., 2019; Zhang \& Zhang, 2017). The entrepreneurial behavior of floating population in strange land has attracted continuous attention.

Floating population entrepreneurship in strange land refers to the behavior of floating population to identify, create and utilize economic opportunities, carry out entrepreneurial activities and achieve enterprise development in their inflow areas. The early research on the floating population entrepreneurship in strange land holds that the floating population generally encounters external discrimination, and their limited educational base, professional skills, and resource endowment even lead them to choose to start a business in retail or low-skilled service industries. With the development of the phenomenon of floating population entrepreneurship in strange land, some scholars have found that floating population is a group with internal heterogeneity, and some of floating population with high education and professional skills has begun to enter knowledgeor technology-intensive industries. Floating population entrepreneurship in strange land has gradually exhibited diversified characteristics.

At present, the research on the floating population entrepreneurship in strange land mainly focuses on the entrepreneurial choices, determinants and performance of entrepreneurship, and has obtained relatively rich theoretical results. However, the existing research still has deficiencies, such as research generalization and conclusion ambiguity, which need to be systematically combed and analyzed. This paper focuses on the main topics of floating population entrepreneurship in strange land, and systematically reviews the research on floating population entrepreneurship in strange land from three aspects: the defini- 
tion and measurement of floating population entrepreneurship in strange land, the determinants of floating population entrepreneurship in strange land, and the results of floating population entrepreneurship in strange land, and expands the direction of future research by combining existing research conclusions and deficiencies.

The following arrangement of this paper is as follows: the second section mainly introduces the definition and measurement of floating population entrepreneurship in strange land, including the definition and measurement of "floating population", "rural floating population", "entrepreneurship" and "floating population entrepreneurship in strange land". The third section mainly introduces the determinants of floating population entrepreneurship in strange land, including the analysis of determinants at the micro level, the meso level and the macro level. The fourth section mainly introduces the results of floating population entrepreneurship in strange land, including the analysis of economic results and non-economic results. The fifth section mainly introduces the shortage of current research and the prospect of future research on floating population entrepreneurship in strange land. The sixth section mainly introduces the research contributions and deficiencies of this paper.

\section{Definition and Measurement of Floating Population Entrepreneurship in Strange Land}

Floating population refers to the adults of child-bearing age who have left the area where their household registration is located and live in strange land for the purpose of working and living ${ }^{1}$.Specifically in statistics, the floating population refers to the population separated by households deducts that in municipal districts. The population separated by households refers to the population whose residence is not consistent with the township street where the household registration is located and who has been away from the household registration place for more than half a year. The population separated by households in a municipal district refers to the population in a municipality or a prefecture-level city inside and between district and district, whose residence and household registration place are not in the same township street ${ }^{2}$. Entrepreneurship is the process of identifying, assessing, and leveraging opportunities to create future goods and services (Shane \& Venkataraman, 2000). Gartner (1988); Low and MacMillan (1988); Shook et al. (2003) consider that creating a new business is the core of entrepreneurship. Parker (2009) argues that entrepreneurship includes self-employment and obtaining independent enterprise ownership. Floating population entrepreneurship in strange land refers to the entrepreneurial behavior of floating population in the inflow areas. In addition to taking the floating population as a whole as the research object (Ning \& Duan, 2017; Ye et al.,

${ }^{1}$ The definition of floating population refers to The Administrative Measures on Family Planning ot Floating Population.

${ }^{2}$ The definition of floating population in statistics refers to The Statistical Bulletin of National Economic and Social Development 2018. 
2018b), a lot of researches on floating population entrepreneurship in strange land in China focus on the urban entrepreneurial behavior of rural floating population (Rui, 2017; Wang \& Feng, 2018; Zhou et al., 2017). The rural floating population is the floating population with an agricultural household registration or from rural areas. It is an important part of the floating population, and it mainly reflects the liability of foreignness of floating population from less developed areas to developed areas.

The existing research on the floating population entrepreneurship in strange land mostly uses quantitative analysis, while a few studies take the Xinhua printing entrepreneurs as the research object for qualitative analysis. In the quantitative research, different scholars on the measurement of floating population and entrepreneurship are slightly different, as follows:

The measurement of floating population: In the most commonly used measurement method, the floating population refers to people who have lived locally for 1 month or more, have non-local (county, city) household registration, and are 16 - 59 years old (Li \& Chen, 2018; Ning \& Duan, 2017; Wei et al., 2016). In addition, there are also studies that limit the floating population to people whose current residence is different from the household registration location at the age of 14 (Ye et al., 2018b).

There are three main ways to measure the rural floating population: First, people with agricultural household registration who live in cities or work in cities (Liu et al., 2019; Zhang \& Liu, 2014). Second, that is floating population with agricultural household registration and engaged in non-agricultural work (Jing et al., 2018). Third, that is floating population with agricultural household registration (Yang et al., 2018).

The measurement of entrepreneurship: Existing research mainly considers that the entrepreneurial behavior of floating population includes self-employment and employer. However, some studies consider that there are many differences between self-employment and employer, and explore the differences between them (Jing et al., 2018; Li \& Chen, 2018; Wang \& Feng, 2018). At the same time, some studies consider more strictly that the entrepreneurial behavior does not include self-employment (Rui, 2017; Ye et al., 2018b). Specific to the measurement, self-employment is mainly measured by whether the type of work is self-employment (Li \& Chen, 2018). In addition to measuring whether the type of work is an employer, employer is measured also by the number of employees (employers with 1 or more employees), and private business owners are considered employers (Jing et al., 2018; Wang \& Feng, 2018).

\section{Determinants of Floating Population Entrepreneurship in Strange Land}

There are many determinants of the floating population entrepreneurship in strange land, and the whole can be divided into the following three aspects, namely the micro level, meso level and macro level. 


\subsection{The Micro Level}

Although floating population generally encounters liability of foreignness, different floating population has different personality characteristics and resource endowments, and these factors affect the entrepreneurial choices and entrepreneurial performance of floating population. The determinants at the micro level mainly include the psychological characteristics and individual characteristics of the floating population.

1) Psychological characteristics

The determinants of psychological characteristics include entrepreneurial self-efficacy, entrepreneurial passion and so on. Entrepreneurial self-efficacy refers to the degree of self-confidence in whether an individual can adapt to entrepreneurial roles and successfully complete various entrepreneurial tasks. In the field of floating population entrepreneurship in strange land, entrepreneurial self-efficacy is subdivided into four aspects: innovation management, resource acquisition, opportunity identification and risk management ( $\mathrm{Pu}$ et al., 2016). Among them, resource acquisition and risk management self-efficacy positively affect the entrepreneurial intention of floating population. Entrepreneurship is a high-risk activity that requires continuous resource input, resource acquisition and risk management self-efficacy promote entrepreneurial intentions by helping floating population maintain an optimistic attitude toward entrepreneurial risks and expand resource acquisition channels actively. Innovation management and opportunity identification self-efficacy had no significant effect on entrepreneurial intentions. Floating population entrepreneurship is mostly concentrated in the fields of low-skilled and low-social status, and is mainly based on imitation and replication. Moreover, the degree of innovation and difficulty of floating population entrepreneurship is relatively low. Therefore, floating population does not need to have high innovation management and opportunity identification capabilities. Entrepreneurial passion refers to the conscious and positive identification of entrepreneurs in participating in entrepreneurial activities (Cardon et al., 2009). High entrepreneurial passion promotes the floating population to actively search for entrepreneurial information, accumulate entrepreneurial capital, and embed the core position of social networks to obtain key resources. And then, it is beneficial for floating population to combine information resources, identify entrepreneurial risks, and develop entrepreneurial opportunities. At the same time, floating population shows high entrepreneurial passion helps to obtain the investment and cooperation of stakeholder and increase legitimacy. Therefore, entrepreneurial passion is positively regulating the positive effects of social network embeddedness on opportunity development and organizational legitimacy, thereby promoting entrepreneurship (Rui, 2017).

2) Individual characteristics

The research on individual characteristics mainly includes demographic characteristics such as gender, age, marital status, household registration, non-cognitive competence, education, work experience, health, and dialect skills. 
Research on demographic characteristics generally considers that male, married, and floating populations with agricultural household registration are more likely to choose a strange land to start a business, and there is an inverted U-shaped relationship between age and entrepreneurship (Liu et al., 2019; Zhu, 2017). But some scholars find some different conclusions. Zhou et al. (2017) found by further subdivision of entrepreneurial types that men are more likely to become entrepreneur (employer), but are not different from women in terms of becoming self-employed. Ning and Duan (2017) found that non-agricultural or local household registration can help reduce financial constraints, improve risk tolerance, and stabilize social security and employment expectations, and that promotes entrepreneurial choice. Liu and Huang (2016) found that floating population with non-agricultural household registration is more inclined to obtain employment rather than entrepreneurship.

Non-cognitive competence refers to a relatively stable way of thinking, feeling and behavior, which reflects the specific response of an individual under certain circumstances. Zhu (2019) constructed non-cognitive competence indicators based on NEO personality characteristic, it is found that the improvement of non-cognitive competence can help floating population to carry out entrepreneurial activities, especially self-employed entrepreneurial activities. Further research on the mechanism of non-cognitive competence on entrepreneurship revealed that non-cognitive competence promotes entrepreneurship by promoting the accumulation of human capital and social capital. Further considering the characteristics of floating population, the study found that non-cognitive competence plays a greater role in promoting the entrepreneurship among women, low skills and floating population from non-first-tier cities. Specific to the non-cognitive competence measurement dimension, the study found that rigorousness and agreeableness promoted entrepreneurial choice, while openness and neuroticism were not conducive to entrepreneurship.

Educational level, work experience, and health level are the more common variable in the study of determinants of floating population entrepreneurship in strange land, and they are often combined into the variable of human capital. Due to the different research samples, definitions of entrepreneurship and divisions of entrepreneurial types, there are three different conclusions about the impact of the above factors on floating population entrepreneurship in strange land. First, there is an inverted U-shaped relationship between education level, work experience and entrepreneurial choice. The floating population with junior and senior high school education level is more inclined to choose entrepreneurship, and the education level of undergraduate and above reduces the entrepreneurial tendency (Jing et al., 2018; Liu et al., 2019). When the floating population has a low level of education or lack of work experience, it does not have the basic skills and learning capabilities for entrepreneurship, and it is difficult to carry out entrepreneurial activities. With the increase of education and work experience, their entrepreneurial intention and entrepreneurial ability increase. 
When the floating population has an undergraduate education level or above, it has more opportunities to obtain stable jobs with high salaries and high social status in the labor market, and its entrepreneurial opportunity costs are higher, so they are more inclined to choose employment than entrepreneurship. When floating population have a long working life, they are less likely to carry out entrepreneurial activities, especially necessity entrepreneurship. Secondly, there is a positive relationship between education level, work experience and entrepreneurial choice, especially being an employer. Educational and work experience are conducive to improving the knowledge level, cultivating entrepreneurial skills, and broadening the social network of the floating population, which in turn help them identify entrepreneurial opportunities and carry out entrepreneurial activities (Jing et al., 2018; Zhou et al., 2017). At the same time, with the increase in education level and working experience, the aspiration of upward mobility and the ability of accepting new things of floating population increase, and they are more willing to start a business (Li, 2018; Zhang \& Liu, 2014). Third, there is a negative relationship between education level and entrepreneurial choice, especially necessity self-employment (Wei et al., 2016; Zhou et al., 2017; Zhu, 2017).

Dialects are the carrier and media of regional culture. Existing studies have found that proficient in local dialects can help reduce the liability of foreignness, so as to promote the floating population to understand local culture, embed in local social network and break down barriers to entrepreneurship (Wei et al., 2016; Yang et al., 2019). At the same time, Wei et al. (2016) found that the promotion effect of dialect level on entrepreneurship is more significant in urban-rural junctions, county towns, rural villages, and inter-dialect regions. Yang et al. (2019) found that the promotion effect of dialect on entrepreneurship is more obvious in areas where there are fewer types of languages and Mandarin is less popular.

\subsection{The Meso Level}

The meso level factors include family level factors and social network factors.

1) Family level

The determinants at the family level mainly include two aspects, material and non-material capital. Family material capital such as household income and cultivated land quantity affect entrepreneurial choices by the initial capital and opportunity cost of entrepreneurship. First, research based on the perspective of capital suggests that family physical capital promotes the floating population entrepreneurship in strange land. The economic base of the family or the area of family cultivated land not only provides initial capital for the floating population, but also strengthens the ability of to resist risks (Zhang \& Liu, 2014). Second, based on the perspective of the opportunity cost of entrepreneurship, research suggests that family material capital reduces the willingness of floating population to start a business in strange land. Because of abandoning vested 
gains and choosing to start over, the cultivated land and housing of the home family represent an opportunity cost. When this value exceeds the income from entrepreneurship, it prevents floating population from starting a business in strange land (Wei et al., 2016; Zhu, 2017).

Family non-material capital such as family origin not only symbolizes economic and social status, but also represents the sum of potential family material capital. Family culture or family entrepreneurial atmosphere affects the entrepreneurial attitude and direction of floating population and having an important impact on the entrepreneurial choice of floating population. Zhou et al. (2017) used the family component as the proxy variable for the intergenerational transmission of entrepreneurship, and explored the impact of the non-material capital brought by the family component on being a self-employed and an employer of floating population in the city. The study found that floating population with a family background of landlords, capitalists, and small craftsmen are more inclined to be an employer. The intergenerational transmission of family non-material capital has an important impact on the entrepreneurial choice of the next generations.

2) Social network

Existing research mainly focuses on social networks based on hometown relations and social networks with local residents in the inflow areas. Among them, hometown relation social networks include social networks established with the same township before migration and individuals with hometown relations in the inflow areas.

Affected by the family tradition, the floating population is embedded in the social basic network based on blood, kinship, and geography; and affected by the rural complex of "old people see old people, two tears", the hometown relation social network in inflow areas can be quickly established and the network participant are willing to help each other and share resources. However, such social networks are homogeneous and closed due to their limited communication scope (Ding, 2012). Therefore, the impact of hometown relation social networks on floating population entrepreneurship in strange land is dualistic. On the one hand, the hometown relation social network is conducive to access to various information and resources in the existing network, expand the access to social capital, and embed in the local social network. For example, floating population can obtain market information and resources and raise economic capital through friends working outside the country; seek relatives who serve as civil servants and teachers for economic, emotional, and policy guidance (Zhang \& Liu, 2014). They also can be embedded local social networks through the social relationships of other outside entrepreneurs and managers, and then obtaining legitimacy. In addition, the size of the floating population also helps floating population to carry out entrepreneurial activities in the local area (Feng et al., 2016). On the other hand, over-embedding hometown relation social networks is not conducive to access to heterogeneous information, and foster creative 
thinking. The emotional stumbling of the hometown relations may also limit the opportunities for floating population to develop other markets. The hometown relation social network is not conducive to or even restricts the entrepreneurial choice of floating population ( $\mathrm{Zhu}, 2017$ ).

The contact with the local residents in inflow areas makes floating population embedded in the local social network. Such network relationships are heterogeneous due to the wide range of communications. The local social network embeddedness is conducive for floating population to obtain local information and resources, establish legitimacy, and often play a positive role in entrepreneurship. For example, getting along well with the local residents is not only beneficial for the floating population to enhance their understanding of the local economy and society, to obtain information and resources related to the local market, but also beneficial for them to obtain entrepreneurial emotional support (Zhang \& Liu, 2014). Mastering the local dialect or living in a community based on local residents help floating population quickly adapt to local culture and establish legitimacy (Zhu, 2017).

Some scholars have explored the different roles played by the hometown relation social network and the local social network on the floating population entrepreneurship in strange land. They consider that although the enhancement of different social networks significantly promotes the floating population entrepreneurship in strange land, the hometown relation social network mainly affects the physical capital investment in the early stage of entrepreneurship and mainly plays a role in entrepreneurial decisions, and the local social network mainly affects the transfer of social capital, and therefore has a greater impact on entrepreneurial success (Yang et al., 2018).

\subsection{The Macro Level}

The macro level determinants mainly include economic environment and institutional environment. Among them, the factors of economic environment mainly include the level of urban development, labor demand, minimum wage standards and the degree of Internet development; the factors of institutional environment mainly include national policies, household registration systems, and the degree of market openness. In addition, some scholars have explored the impact of the economic and institutional distance between the inflow and outflow areas on the floating population entrepreneurship in strange land.

1) Economic environment

China's floating population usually moves from low-economic-level areas to high-economic-level areas. The size and development level of cities is crucial to the decision-making of employment and entrepreneurship of the floating population. Although there are many entrepreneurial opportunities in economically developed areas, some studies have also found that floating population pay attention to general employment opportunities in economically developed areas, and they are more inclined to choose employment than entrepreneurship (Feng 
et al., 2016). Similarly, the greater the regional labor demand are, the more employment opportunities that can be provided to the floating population, and the lower intention to start a business.

Floating population usually faces employment discrimination in the labor market, and it is difficult to obtain considerable wage income. The increase of their income depends largely on the minimum wage standard. The higher the minimum wage is, the more possibility for floating population to achieve income growth through employment, so they are more likely to choose employment than entrepreneurship (Li \& Chen, 2018). At the same time, the minimum wage standard reflects the level of social security in the region to a certain extent. When the level of regional social security is higher, the floating population has more ways to make a living, and the entrepreneurial intention decreases (Ning \& Duan, 2017). However, Li and Chen (2018) also pointed out that as the gender, human capital, economic capital, and entrepreneurial region of the floating population are different, the minimum wage standard may plays a different role in their entrepreneurial choice.

The degree of Internet development affects the acquisition of market information and the accumulation of social capital of floating population. The higher the degree of Internet development, the more channels the floating population can obtain market information, so as to grasp the opportunity of entrepreneurship in time. At the same time, the development of the Internet makes it more convenient for people to communicate with each other, which not only helps the floating population to maintain the existing social network, but also promotes them to expand new social relations. Therefore, the use of the Internet by floating population is conducive to their entrepreneurial activities (Zhang et al., 2019).

2) Institutional environment

Existing studies have found that the lack of policy support is an important environmental factor restricting the entrepreneurship of floating population. The existing policies mainly protect the interests of local residents, the floating population especially that without a local household registration in the face of complicated business administrative approval procedures, and it is difficult for them to obtain financial support through formal channels such as government support, bank loans and venture investment. The improvement of the policy environment will help reduce the difficulty of the floating population in entrepreneurship and promote their entrepreneurial choice (Feng et al, 2016; Zhu, 2014).

China's household registration system is an important cause of discrimination against floating population and an important factor restricting floating population entrepreneurship. Floating population usually has difficulty in obtaining local household registration. The higher the threshold for regional settlement, the higher the gold content of the household registration, and the more discrimination and restrictions the floating population encounters. For example, it is difficult for floating population to establish their local identity psychologically, 
and their sense of belonging to the region is low. Floating population does not enjoy equal social welfare policies and security systems, and their living cost is high. It is difficult for the floating population to integrate into the local society, and the transaction cost in entrepreneurship is high (Feng et al., 2016; Li, 2019).

In addition, some scholars have explored the impact of economic and institutional distances between the inflow and outflow areas on floating population entrepreneurship in strange land (Ye et al., 2018b), and the study have found that the larger economic distance promotes the floating population entrepreneurship in strange land by providing a larger consumer market, rich entrepreneurial opportunities and strong knowledge spillover effects; the larger institutional distance promotes the floating population entrepreneurship in strange land by providing more entrepreneurial resources and opportunities, promoting the fair allocation of information and resources and entrepreneurial opportunities.

\section{Results of Floating Population Entrepreneurship in Strange Land}

The existing research on the results of floating population entrepreneurship in strange land is mainly based on economic and non-economic perspectives. Economic results mainly include individual level, enterprise level and social level, and non-economic results mainly include social identity, social integration, entrepreneurial happiness and other subjective feelings.

\subsection{Economic Results}

1) Individual level

At the individual level, the economic results mainly focus on the income of floating population entrepreneurs. Previous studies have found that there is no significant difference between the employment level of floating population entrepreneurs and local enterprises, and their entrepreneurial income is even significantly higher than that of local entrepreneurs (Zhang \& Zhang, 2017). Urban household registration, local household registration and public services help to increase the entrepreneurial income of floating population (Ning \& Duan, 2017).

2) Enterprise level

Economic results at the enterprise level mainly focus on the entrepreneurial success and enterprise performance of the floating population.

Existing studies have explored the entrepreneurial success of floating population through the indicator of whether there is a fixed business place (Yang et al., 2018), and found that local social network embeddedness is an effective way to overcome entrepreneurial difficulties and achieve entrepreneurial success. The exit rate of enterprises of floating population is even significantly lower than that of local enterprises (Liu et al., 2019).

Existing studies have explored the entrepreneurial performance of floating population from two aspects: entrepreneurial input and entrepreneurial output. Among them, the indicators of entrepreneurial investment include initial in- 
vestment, the area of fixed production and operation sites, the handling of relevant business licenses, the number of employees, and technical input, etc. The indicators of entrepreneurial output include profitability, total assets, and the duration of the enterprise, etc. (Liu et al., 2019; Yang, 2016; Yang et al., 2018). Although some studies have found that there are the characteristics of low technical threshold, small scale, short start-up period, low initial investment and low profit level in floating population entrepreneurship (Yang et al., 2018), some studies have found that the entrepreneurial performance of floating population is comparable to that of local residents (Liu et al., 2019), and human capital and social capital can help improve the entrepreneurial performance of floating population (Yang, 2016).

3) Social level

At the social level, the economic results mainly focus on the clustering phenomenon of floating population entrepreneurship and its impact on the economic development, innovation and entrepreneurship of the city.

The research on the phenomenon of entrepreneurial clustering of floating population entrepreneurship has found that although the entrepreneurial clustering reduces the difficulty of entrepreneurship, it restricts the long-term development of enterprises. Existing studies have explored the causes of entrepreneurial clustering of floating population in cities from the perspectives of psychology and social capital (Pu et al., 2016), the study found that the innovation of floating population entrepreneurship is low, and it mainly based on copy and imitate. The single social network embeddedness and the low self-efficacy of entrepreneurship result in the formation of the phenomenon of low-level urban entrepreneurship clustering.

Existing studies have found that the size of floating population in cities significantly promotes urban entrepreneurial activity, and the degree of regional marketization and the level of Internet development further promote the influence of floating population on the entrepreneurial activity of cities (Ye et al., 2018a). At the same time, some scholars have found that although the entrepreneurship of floating population is mainly concentrated in the wholesale, retail and food industries, it has experienced rapid growth in high-end services such as finance, real estate and social welfare, and the floating population entrepreneurship is gradually diversified and specialized (Liu et al., 2019).

\subsection{Non-Economic Results}

The non-economic outcomes of floating population entrepreneurship in strange land are mainly concerned with the subjective psychological feelings of floating population, including social identity, social integration and entrepreneurial happiness.

Entrepreneurship of floating population in strange land promotes deconstruction of the identity of external population and improves urban adaptability (Yang, 2011). The entrepreneurship of floating population in cities increases their economic income, promotes their embeddedness of local social networks, 
and improves their economic and social status, which is conducive to the acceptance of local culture and the active integration into the local society.

The social integration of floating population has the characteristics of "low level" and "non-equilibrium". Liu (2013) has subdivided social integration into economic integration, community integration, social relationship integration, and psychological and cultural integration, and has found that various types of social integration among floating population are generally low, and there are unbalanced, especially floating population is rarely willing to take the initiative incorporating into the local society, and the institutional and cultural environment, migration patterns of floating populations, and business models affect their social integration. However, some studies suggest that urban entrepreneurship of floating population is not only a process of their own integration into the local society, but also has the characteristics of intergenerational transmission (Sun, 2018).

Existing studies have explored the entrepreneurial happiness of floating population from the dimensions of social status, work and life satisfaction, and value realization. Studies have found that the entrepreneurial happiness of floating population is at a medium level (Rui, 2017). The new generation of floating population attaches particular importance to the local social network embeddedness and opportunity development, and it is easier to obtain happiness from innovative entrepreneurial behavior.

\section{Inadequate research and Future Prospects}

At present, the research on floating population entrepreneurship in strange land in China has achieved rich theoretical results. However, the subdivision of floating population entrepreneurship in strange land, the interaction of determinants of floating population entrepreneurship in strange land, the process of floating population entrepreneurship in strange land, and the entrepreneurial performance of floating population in strange land need to be further deepened.

\subsection{Subdivision of Floating Population Entrepreneurship Types}

The current research on the floating population entrepreneurship in strange land mainly focuses on the entrepreneurial behavior of general floating population in strange land and the urban entrepreneurial behavior of rural floating population, and pays less attention to the phenomenon of floating population from urban areas. At the same time, it ignores the motivation and mechanism of entrepreneurship that floating population from more developed areas to less developed areas. Compared with rural floating population, the economic and education level of floating population from urban areas are relatively high. Do they face lower restrictions and obstacles in entrepreneurship, and what is the difference between their entrepreneurial motivations and behaviors? Future research can explore the entrepreneurial behavior of urban floating population in strange land. In addition, although the economically underdeveloped regions seem to 
have fewer entrepreneurial opportunities, many emerging industries are waiting to rise in these regions. In order to grasp the first-mover advantage of the market, companies or individuals with economic strength are willing to turn to less-developed regions for seeking entrepreneurial opportunities. Therefore, future research can focus on the entrepreneurial behavior of floating population from more developed areas to less developed areas.

\subsection{The Interaction of Determinants of Floating Population Entrepreneurship in Strange Land}

Although the existing studies have explored the determinants of floating population entrepreneurship in strange land from different levels, few studies have focused on the interaction between different factors. The entrepreneurial behavior of the floating population is the result of multiple factors, and there may be mutual substitution or coordination between different factors. For example, hometown relation social network can make up for the lack of economic or human capital of the floating population, and then promote their entrepreneurial activities. Regional discrimination or imperfect institutional environment hinders the entrepreneurial activities of the floating population, but the floating population with higher professional skills and resource endowment are relatively less affected. Future research can focus on the interaction between different factors on the impact of floating population entrepreneurship in strange land.

\subsection{Research on the Process of Floating Population Entrepreneurship in Strange Land}

Entrepreneurship is a process of continuous resource input and strategic planning. Existing studies pay more attention to the determinants and results of floating population entrepreneurship, and the process of entrepreneurship needs to be improved. Through literature review, we have found that the floating population is a group with internal heterogeneity, and different floating population has different personality characteristics and resource endowments, and their initial entrepreneurial strategy choice and enterprise development direction are also different. For example, floating population with lower education level and economic capital usually choose to enter retail or low-skilled service industries, and adopt low-cost strategies or centralized strategies, while floating population with higher education level and professional skills can choose to enter high-tech industries such as finance and artificial intelligence, and adopt differentiation strategies. Future research can focus on the entrepreneurial process, the development and strategic transformation of enterprises after that.

\subsection{Entrepreneurial Performance of Floating Population in Strange Land}

There is no uniform conclusion about the existing research on the entrepreneurial performance of floating population in strange land, the reason is that different studies select different samples of floating population, and the defini- 
tion and measurement of entrepreneurship and enterprise performance are different. At the same time, few scholars have conducted comparative studies on different samples of floating population, types and areas of entrepreneurship. Future research can focus on different types of floating population entrepreneurship behavior, and conduct comparative studies on them according to their different entrepreneurial characteristics, so as to reveal the entrepreneurial performance of floating population more accurately.

\section{Conclusion}

With the development of urbanization in China, the floating population has become an important driving force for urban economic development. This paper focuses on the main theoretical results of the research on floating population entrepreneurship in strange land, systematically combs and reviews the research on floating population entrepreneurship in strange land from three aspects: the definition and measurement of floating population entrepreneurship in strange land, the determinants of floating population entrepreneurship in strange land, and the results of floating population entrepreneurship in strange land. And then, based on the existing analysis conclusions and deficiencies, this paper expands the future research direction. This paper considers that the subdivision of types of floating population entrepreneurship in strange land, the interaction of determinants of floating population entrepreneurship in strange land, the process research of floating population entrepreneurship in strange land, and the entrepreneurial performance of floating population in strange land need to be further deepened.

This paper has the following theoretical contributions. Firstly, this paper systematically sorts out and integrates the main research contents of the floating population entrepreneurship in strange land under the Chinese context, and reveals the development context and development status of this topic. Although there are abundant theoretical achievements in the existing studies on floating population entrepreneurship in strange land, there are still some deficiencies such as research generalization and conclusion ambiguity, and few studies have systematically sorted out and reviewed them. This paper clearly defines the connotation of floating population entrepreneurship in strange land and standardizes it from the perspective of measurement. And then, based on the existing main research topics, this paper classifies and integrates the research contents of floating population entrepreneurship in strange land from the perspective of entrepreneurial process, and clearly shows the research development context and research development stages of floating population entrepreneurship in strange land. At the same time, it is also beneficial to compare and analyze the existing research conclusions, and clarify the differences between different research conclusions. Secondly, based on the existing research conclusions and deficiencies, this paper expands the research development direction of the floating population entrepreneurship in strange land. Existing research topics 
and research content on floating population entrepreneurship in strange land are gradually unified, and lack of innovative research. By integrating the existing research content, we have not only expanded the development direction of the existing main research topics, but also expanded new research topics, further promoting the diversified research of floating population entrepreneurship in strange land.

At the same time, this paper also has the following research deficiencies. Firstly, although this paper strictly refers to the current mainstream research methods when selecting research samples and carefully reads the literature one by one, this paper is completed by the author alone, and it is inevitable that there will be omissions when collating the existing literature. In order to reduce the impact caused by the above situation, the authors searched the literature through similar keywords as much as possible, and carefully checked the disputed literature by reading the text of the literature. After determining the research samples, the author read the literature one by one and consulted other teachers and classmates when in doubt to try to ensure the accuracy of the understanding. Secondly, although this study systematically combs and integrates the research on the floating population entrepreneurship in strange land, and expands the future research direction, it does not build a systematic analysis framework. Future reviews can combine existing research and future prospects to build a systematic research framework in order to more clearly show the research logic of floating population entrepreneurship in strange land.

\section{Acknowledgements}

The successful publication of this paper benefits from the teachers' careful instruction and classmates' selfless help. Here I am very grateful to the teachers and classmates who gave valuable opinions on this paper. At the same time, I am also very grateful to Jinan University for the challenges and opportunities brought to me by the three-year postgraduate program. I will work harder and strive for continuous progress on the path of academic research.

\section{Conflicts of Interest}

The author declares no conflicts of interest regarding the publication of this paper.

\section{References}

Cardon, M. S., Wincent, J., Singh J., \& Drnovsek, M. (2009). The Nature and Experience of Entrepreneurial Passion. Academy of Management Review, 34, 511-532. https://doi.org/10.5465/amr.2009.40633190

Ding, G. J. (2012). Research on the Impact of Social Capital, Opportunity Recognition to Peasants Entrepreneurial Performance. Hangzhou: Zhejiang University.

Feng, J. X., Tang, S. S., \& Yang, Z. S. (2016). Determinants of Entrepreneurial Behavior of Rural Migrants in Urban Society: From the Perspective of "Human-Environment Relationship”. Geographical Research, 35, 148-162. 
Gartner, W. B. (1988). “Who Is an Entrepreneur?” Is the Wrong Question. American Journal of Small Business, 12, 11-32. https://doi.org/10.1177/104225878801200401

Jing, Z. F., Chen, J. J., \& Yang, X. L. (2018). Self-Employed or Employed: Mechanism and Empirical Test of Human Capital in Rural Floating Population-Analysis of Experience Based on CGSS Data. Issues in Agricultural Economy, No. 6, 87-97.

Li, J., \& Chen, Y. L. (2018). The Impact of the Minimum Wage on Entrepreneurship-Study Based on National Floating Population Dynamic Survey Data. Review of Industrial Economics, No. 2, 105-124.

Li, J. (2018). Influence of Human Capital on Entrepreneurial Process of Migrant Workers in Urban China. Journal of South China Agricultural University (Social Science Edition), 17, 42-51.

Li, X. B. (2019). City Settlement Threshold and Migrant's Entrepreneurial Choice: Analysis Based on the Micro Data from 37 Large and Medium Cities in China. Nanjing: Nanjing University.

Liu, C. Y., Ye, L., \& Feng, B. (2019). Migrant Entrepreneurship in China: Entrepreneurial Transition and Firm Performance. Small Business Economics, 52, 681-696.

https://doi.org/10.1007/s11187-017-9979-y

Liu, C. Y., \& Huang, X. (2016). The Rise of Urban Entrepreneurs in China: Capital Endowments and Entry Dynamics. Growth and Change, 47, 32-52. https://doi.org/10.1111/grow.12117

Liu, Y. (2013). The Social Integration of Business-Oriented Migrant Farmers-A Case Study of Xinhua Printing Farmers in Hunan Province. Jinhua: Zhejiang Normal University.

Low, M. B., \& MacMillan, I. C. (1988). Entrepreneurship: Past Research and Future Challenges. Journal of Management, 14, 139-161. https://doi.org/10.1177/014920638801400202

Ning, G. J., \& Duan, L. L. (2017). Migrant's Self-Employment Choice and Income: The Role of Household Registration System and Reform Implication. China Economic Quarterly, 16, 771-792.

Parker, S. C. (2009). The Economics of Entrepreneurship. Cambridge: Cambridge University Press. https://doi.org/10.1017/CBO9780511817441

Pu, M. Z., Zheng, F. T., \& Fu, J. H. (2016). Rural Migrants' Self-Employment Cluster in Urban China: From Perspective of Entrepreneurial Self-Efficacy. Journal of Nanjing Agricultural University (Social Sciences Edition), 16, 61-76.

Rui, Z. Y. (2017). Unleash Entrepreneurial Passion: The Impact of Urban Social Networks Embeddedness on Migrant Workers' Entrepreneurial Happiness in the City. Modern Economic Science, 39, 25-32.

Shane, S., \& Venkataraman, S. (2000). The Promise of Entrepreneurship as a Field of Research. The Academy of Management Review, 25, 217-226.

https://doi.org/10.5465/amr.2000.2791611

Shook, C. L., Priem, R. L., \& McGee, J. E. (2003). Venture Creation and the Enterprising Individual: A Review and Synthesis. Journal of Management, 29, 379-399. https://doi.org/10.1016/S0149-2063_03_00016-3

Sun, W. Z. (2018). Migrant Workers' Integration into Urban Society from Life Experience Perspective. Journal of Shenzhen University (Humanities \& Social Sciences), 35, 111-120.

Wang, C. C., \& Feng, D. W. (2018). The Determination Mechanism of Entrepreneurial Behavior for Chinese Rural-Urban Immigrants: Based on the Analysis Perspective of 
Social Networks. China Economic Quarterly, 17, 355-382.

Wei, X. H., Chen, S. Y., \& Li, J. H. (2016). Floating Population's Dialect Skill and Entrepreneurial Choice. Chinese Journal of Population Science, No. 6, 36-46.

Yang, J. (2016). The Research of Effects of Human Capital on Migrant Workers' Urban Entrepreneurial Performance. Shanghai: East China University of Political Science and Law.

Yang, J. L. (2011). Research on the Identification of the Independent Business Peasant Labourers in Chinese City-A Case from a City in Shandong Province. Nanjing: Nanjing University of Aeronautics and Astronautics.

Yang, X. Y., Pan, Y., \& Tong, X. L. (2018). Bilateral Social Network and Remote Entrepreneurship of Rural Households-Evidence from 2527 Rural Migrants in Seven Cities of China. Journal of Agrotechnical Economics, No. 9, 30-41.

Yang, Y., Zhu, C., \& Tan, Y. (2019). Dialect Competence, Language Environment and Urban Migration Entrepreneurship. Chinese Journal of Sociology, 39, 211-236.

Ye, W. P., Li, X. C., \& Chen, Q. Y. (2018a). How Immigrant Populations Affect City Entrepreneurship Activity: Mechanisms and Evidence. Economic Research Journal, 53, 157-170.

Ye, W. P., Li, X. C., \& Zhu, H. (2018b). Entrepreneurship in Strange Land and Embeddedness of Social Networks. Management World, 34, 139-156.

Zhang, G. S., \& Liu, Y. H. (2014). Research on the Influence of Human Capital and Social Capital on the New Generation of Migrant Workers' Entrepreneurial Employment-Based on the Investigation of Three Cities in Liaoning Province. Journal of Agrotechnical Economics, No. 6, 4-13.

Zhang, J., Zhou, X. Q., \& Xiao, S. S. (2019). From Migration to Entrepreneurship: More Discussion on the Effect of the Internet Use on Entrepreneurship. Journal of Chongqing University (Social Science Edition), 1-16.

Zhang, L. Q. (2016). The Impact of Family Migration on the Employment Status of Floating Population-Based on the Analysis of the 2013 Floating Population Dynamic Monitoring Survey Data. Fuzhou: Fujian Normal University.

Zhang, S. Z., \& Zhang, L. P. (2017). The Effect of Floating Population in China on Entrepreneurship. Human Resources Development of China, No. 7, 138-148.

Zhou, M. H., Jean-Louis, A., \& Tao, R. (2017). Intergenerational Transmission of Entrepreneurship among Chinese Migrants. Economic Research Journal, 52, 74-87. https://doi.org/10.1016/j.iref.2017.01.018

Zhu, Y. T. (2014). The Mechanism Research of Migrant Workers' Urban Entrepreneurial Level and Policy Support: Based on the Case Study of East China. Shanghai: East China University of Political Science and Law.

Zhu, Z. S. (2017). Research on the Rural Migrant Worker's Behavior of Choosing the Self-Employment in Urban China. Beijing: Capital University of Economics and Business.

Zhu, Z. S. (2019). Non-Cognitive Competence and Rural Migrant Entrepreneurship-Empirical Evidence from CMDS. Human Resources Development of China, 36, 93-107. 